\title{
Health Technology Assessment: confronto tra HD ed HDF on-line secondo i QALYs
}

\author{
U. Tulli ${ }^{1}$, E. Valeri ${ }^{2}$
}

${ }^{1}$ Infermiere Coordinatore, UOC Nefrologia e Dialisi Ospedale di Tivoli, Roma

${ }^{2}$ Infermiera Specializzata, UOC Nefrologia e Dialisi Ospedale di Tivoli, Roma

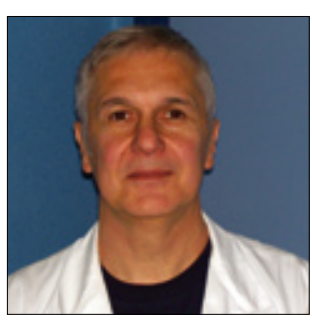

Umberto Tulli

\section{Introduzione}

Il nostro sistema sanitario (SSN) si trova ad affrontare il problema della soddisfazione di crescenti bisogni assistenziali con una quantità di risorse disponibili limitata. Tale situazione implica la necessità di valutare l'appropriatezza delle procedure diagnostico-terapeutiche, delle tecnologie da utilizzare nell'assistenza, con l'obiettivo di individuare l'allocazione ottimale delle risorse disponibili.

L'Health Technology Assessment è un approccio multidimensionale per l'analisi delle implicazioni medico-cliniche, organizzative, economiche ed etiche di una tecnologia attraverso la valutazione di più fattori quali l'efficacia, la sicurezza, i costi, l'impatto sociale e organizzativo.

\section{Materiali e Metodi}

Lo studio è stato condotto presso l'Unità Operativa Complessa di Nefrologia e Dialisi dell'Ospedale Civile S. Giovanni Evangelista di Tivoli (Roma) e presso l'UDD (Unità Dialitica Decentrata) c/o Italian Hospital Group SpA di Guidonia, ed è stato effettuato nel periodo gennaio-ottobre 2010 .

I pazienti oggetto dello studio a Tivoli sono stati 28 , mentre a Guidonia sono stati 82 , il $90 \%$ ha riconsegnato il questionario.

Lo strumento utilizzato è stato il: Questionario SF-36 (strumento multi-dimensionale che consta di 8 dimensioni e di 11 domande) (9).

Per i Risultati consultare la Tabella I alla pagina seguente.

\section{ACU: analisi costo/utilità}

Tecnica che massimizza il rapporto utilità/costo (o minimizza il rapporto costo/utilità) due alternative (interventi tecnici) in cui l'utilità è espressa in termini fisici e, in particolare, in termini di QALYs (quality adjusted life years) cioè anni di vita guadagnati, grazie all'intervento, ponderati per la qualità della vita. In base alla ricerca bibliografica $(1,4,7,8)$ abbiamo scelto di utilizzare l'ACU per due diverse tecniche dialitiche: emodialisi standard HD con una tariffa regionale (Lazio) di $€ 171,60$ a seduta; emodiafiltrazione HDF con una tariffa regionale (Lazio) di $€$ 208,96 a seduta.

\section{Risultati}

Lo studio è stato condotto su 42 pazienti suddivisi su due gruppi (21 per gruppo), omogenei per età anagrafica ed età dialitica, appartenenti al Centro Dialisi di Guidonia; inoltre abbiamo preso in considerazione due dati oggettivi: il KT/V (efficienza dialitica) e l'Hb (emoglobina) (Tab. II). 


\section{TABELLA I}

\section{QUESTIONARIO SULLO STATO DI SALUTE SF-36}

\begin{tabular}{|c|c|c|}
\hline Domanda & U.D.D. GUIDONIA & P.O. TIVOLI \\
\hline 1) In generale direbbe che la Sua salute è: & $36 \%$ scadente & $25 \%$ scadente \\
\hline $\begin{array}{l}\text { 2) Come giudica la sua salute rispetto a un } \\
\text { anno fa: }\end{array}$ & $36 \%$ decisamente peggiore & $25 \%$ decisamente peggiore \\
\hline 3) La sua salute limita delle attività? & $\begin{array}{l}63 \% \text { limita molto attività pesanti } \\
10 \% \text { attività moderate }\end{array}$ & $\begin{array}{l}70 \% \text { limita molto attività pesanti } \\
24 \% \text { attività moderate }\end{array}$ \\
\hline $\begin{array}{l}\text { 4) nell'ultimo mese ha riscontrato problemi } \\
\text { sul lavoro o nelle attività quotidiane } \\
\text { a causa della sua salute fisica? }\end{array}$ & $\begin{array}{l}60 \% \text { trova difficoltà nello svolgimento } \\
\text { delle attività lavorative quotidiane }\end{array}$ & $\begin{array}{l}80 \% \text { trova difficoltà nello svolgimento } \\
\text { delle attività lavorative quotidiane }\end{array}$ \\
\hline $\begin{array}{l}\text { 5) Nell'ultimo mese ha riscontrato problemi } \\
\text { sul lavoro o nelle altre attività a causa } \\
\text { del suo stato emotivo? }\end{array}$ & $\begin{array}{l}56 \% \text { a causa del proprio stato emotivo trova } \\
\text { difficoltà nello svolgimento delle attività } \\
\text { lavorative quotidiane }\end{array}$ & $\begin{array}{l}75 \% \text { a causa del proprio stato emotivo trova } \\
\text { difficoltà nello svolgimento delle attività } \\
\text { lavorative quotidiane }\end{array}$ \\
\hline $\begin{array}{l}\text { 6) Nell'ultimo mese lo stato di salute e lo stato } \\
\text { emotivo hanno interferito con attività sociali? } \\
\text { (famiglia, amici ecc.) }\end{array}$ & $\begin{array}{l}54 \% \text { afferma che il proprio stato di salute } \\
\text { ed emotivo non interferisce con le normali } \\
\text { attività sociali }\end{array}$ & $\begin{array}{l}40 \% \text { afferma che il proprio stato di salute } \\
\text { ed emotivo non interferisce con le normali } \\
\text { attività sociali }\end{array}$ \\
\hline $\begin{array}{l}\text { 7) Quanto dolore fisico ha provato nell'ultimo } \\
\text { mese? }\end{array}$ & $\begin{array}{l}48 \% \text { afferma che il dolore percepito è molto } \\
\text { lieve }\end{array}$ & $35 \%$ afferma che il dolore percepito è forte \\
\hline $\begin{array}{l}\text { 8) In che misura il dolore l'ha ostacolata sia } \\
\text { in casa sia fuori? }\end{array}$ & $\begin{array}{l}48 \% \text { afferma che il dolore percepito non } \\
\text { interferisce nello svolgimento delle normali } \\
\text { attività quotidiane }\end{array}$ & $\begin{array}{l}35 \% \text { afferma che il dolore percepito è forte } \\
\text { tanto da ostacolare le normali attività } \\
\text { lavorative }\end{array}$ \\
\hline $\begin{array}{l}\text { 9) Come si è sentito nell'ultimo mese? } \\
\text { 10) Per quanto tempo il suo stato di salute } \\
\text { ed emotivo hanno interferito nelle sue } \\
\text { attività sociali? }\end{array}$ & $\begin{array}{l}\text { Alle domande nn. } 9 \text { e } 10 \text { il } 47 \% \text { non è mai vivace } \\
\text { e brillante; il } 29 \% \text { si sente sempre molto agitato; } \\
\text { il } 33 \% \text { si sente sempre giù di morale; } \\
\text { il } 16 \% \text { si sente quasi mai calmo e sereno; } \\
\text { il } 37 \% \text { non è mai pieno di energia; il 37\% è } \\
\text { sempre scoraggiato e triste; il } 49 \% \text { si sente } \\
\text { sempre sfinito; il } 33 \% \text { non è mai felice } \\
\text { ed il } 41 \% \text { si sente sempre stanco }\end{array}$ & $\begin{array}{l}\text { Alle domande nn. } 9 \text { e } 10 \text { il } 47 \% \text { non è mai } \\
\text { vivace e brillante; il } 52 \% \text { è sempre agitato; } \\
\text { il } 29 \% \text { si sente molto giù di morale; il } 44 \% \text { non } \\
\text { è mai calmo e sereno; il } 59 \% \text { non si sente mai } \\
\text { pieno di energia; il } 37 \% \text { è sempre scoraggiato } \\
\text { e triste; il } 46 \% \text { si sente sempre sfinito; il } 24 \% \\
\text { non è mai felice; il } 75 \% \text { è sempre stanco }\end{array}$ \\
\hline $\begin{array}{l}\text { 11) Scelga la risposta che meglio descrive } \\
\text { quanto siano vere o false le seguenti } \\
\text { affermazioni: mi ammalo più facilmente } \\
\text { degli altri; la mia salute è come quella } \\
\text { degli altri; miaspetto che la miasalute andrà } \\
\text { peggiorando; di ottima salute }\end{array}$ & $\begin{array}{l}\text { Al } 25 \% \text { sembra di ammalarsi più facilmente } \\
\text { degli altri; al } 54 \% \text { sembra che la propria salute } \\
\text { non sia affatto come quella degli altri; il } 48 \% \text { si } \\
\text { aspetta che la propria salute andrà peggiorando } \\
\text { mentre il } 77 \% \text { ritiene certamente falso che goda } \\
\text { di ottima salute }\end{array}$ & $\begin{array}{l}\text { Al 28\% sembra di ammalarsi più facilmente } \\
\text { degli altri; il } 64 \% \text { percepisce la propria salute } \\
\text { come quella degli altri; il 53\% si aspetta che } \\
\text { la propria salute andrà peggiorando; mentre } \\
\text { l'86\% ritiene certamente falso che goda } \\
\text { di ottima salute }\end{array}$ \\
\hline
\end{tabular}

TABELLA II - ACU

\begin{tabular}{|l|c|c|c|c|c|}
\hline Pazienti & Età & Età dialitica & Metodica & $\mathrm{Kt} / \mathrm{V}$ & $\mathrm{Hb}$ \\
\hline- & Media & Media & - & Medio & Media \\
\hline 21 & 65,3 & 3 & HD & 1.01 & 10,9 \\
\hline 21 & 64.5 & 2.8 & $\begin{array}{c}\text { HDF- } \\
\text { On line }\end{array}$ & 1,23 & 12,8 \\
\hline
\end{tabular}

\section{Conclusioni}

Il modello dei pazienti in emodialisi è d'interesse particolare per numerose ragioni, soprattutto per la possibilità di verifiche trasversali in ambienti diversi e longitudinali, nel tempo con pazienti che richiedono terapie abbastanza omogenee. Questi pazienti di solito fanno riferimento da molti anni alle stesse strutture, agli stessi Medici e agli stessi Infermieri. Per le malattie croniche, le possibilità terapeutiche inducono un effetto congiunto sia sullincremento di vita attesa sia sul miglioramento della qualità della vita $(5,6)$. Occorre un indicatore in grado di cogliere entrambi gli aspetti: riduzione della morbilità (guadagno in qualità “come vivrò?”) e riduzione della mortalità (guadagno in quantità “quanto vivrò?”) $(2,3,5)$. I benefici conseguiti sono espressi in termini d'aspettativa di vita modificata (ponderata) in funzione della qualità della vita: QALYs (Quality Adjusted Life Years). Il valore attribuito alla qualità della vita (Tab. III) in un dato stato di salute va da " 0 " (morte), (0,53 per l'emodialisi ospedaliera, 0,84 per il trapianto renale) a " 1 " (perfetta salute). 


\begin{tabular}{|l|c|}
\hline Stato di salute & Utilità \\
\hline Buona salute & 1,00 \\
\hline Effetti collaterali trattamento ipertensione & $0,95-0,99$ \\
\hline Angina lieve & 0,90 \\
\hline Trapianto di rene & 0,84 \\
\hline Angina moderata & 0,70 \\
\hline $\begin{array}{l}\text { Alcune limitazioni fisiche e di ruolo sociale } \\
\text { con dolore occasionale }\end{array}$ & 0,67 \\
\hline Dialisi ospedaliera & 0,59 \\
\hline Angina grave & 0,50 \\
\hline $\begin{array}{l}\text { Ansia, depressione e solitudine per la maggior } \\
\text { parte del tempo }\end{array}$ & 0,45 \\
\hline Cieco o sordo o muto & 0,39 \\
\hline Costretto in un letto di ospedale & 0,33 \\
\hline Morto & 0,00 \\
\hline Paraplegico, cieco e depresso & $<0,00$ \\
\hline Costretto a letto con forte dolore & $<0,00$ \\
\hline Modificata da Torrance, 1987 & \\
\hline
\end{tabular}

Osservando i dati emersi dallo studio ci siamo resi conto che i due gruppi percepiscono la qualità della loro vita in maniera molto simile (Fig. 1), nonostante i parametri dialitici ed ematologici, oggetto di studio, siano significativamente migliori nel gruppo in trattamento dialitico con la metodica dell'Emodiafiltrazione. Entrambi i gruppi riferiscono che a causa del proprio stato di salute trovano difficoltà nello svolgimento delle normali attività lavorative quotidiane ( $80 \%$ vs $79 \%)$; si sentono sempre agitati $(52 \%)$, stanchi ( $75 \%$ vs $72 \%$ ) e il $53 \%$ vs $50 \%$ si aspetta che la propria vita andrà peggiorando.

Dalle risposte date dai pazienti sembrerebbe ingiustificato il costo della tecnica on-line vs l'HD standard; ci troviamo di fronte a due dati contrapposti:

- Dato oggettivo: l'efficienza dialitica (KT/V) e l'emoglobina $(\mathrm{Hb})$ del gruppo on-line è mediamente migliore del gruppo HD.

- Dato soggettivo: la qualità di vita percepita dai due gruppi è pressoché sovrapponibile.

Nella valutazione finale che cosa dobbiamo prendere maggiormente in considerazione? Ciò che avverte il paziente 0 quello che ci dicono le analisi e la clinica?

Evidentemente ciò che rende mediocre la qualità di vita percepita dal paziente, qualunque sia la tecnica e i dati clinici, è la consapevolezza che si è dipendenti dalla macchina e dal Centro Dialisi, che la mortalità in dialisi è elevata, che solo il trapianto, per chi è in lista, può sollevarli da questo stato.

Come operatori sanitari dobbiamo tener conto maggiormente dei dati clinici, che oggettivamente fanno preferire

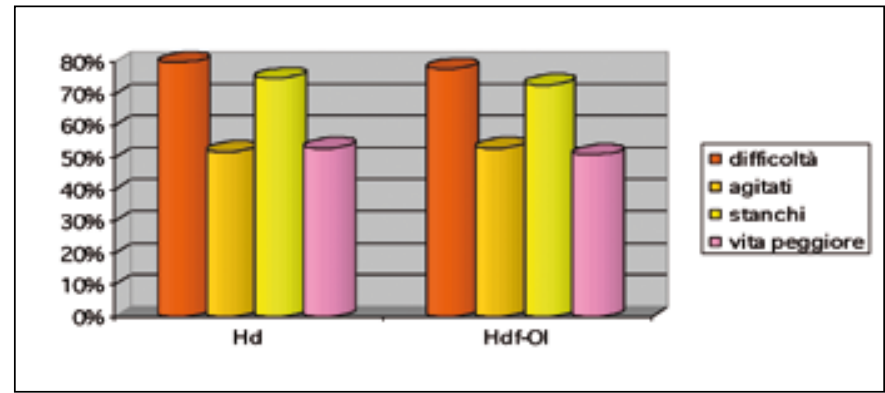

Figura 1 - Qualità della vita percepita dai pazienti.

la tecnica on-line alla metodica classica, ma sarebbe opportuno essere affiancati da delle figure specifiche (per esempio, Psicologo) per poter migliorare lo stato emotivo e psicologico del paziente dializzato e di conseguenza rendere migliore la qualità percepita dal paziente stesso.

\section{Riassunto}

Lo scopo di questo studio è valutare la qualità di vita percepita dalle persone sottoposte a terapia dialitica in rapporto al costo-utilità;

I pazienti oggetto dello studio a Tivoli sono stati 28 , mentre a Guidonia sono stati 82 .

In base alla ricerca bibliografica abbiamo scelto di utilizzare l'ACU (analisi costo/utilità), tecnica che massimizza il rapporto utilità/costo, due alternative (interventi tecnici) in cui l'utilità è espressa in termini fisici e, in particolare, in termini di QALYs (quality adjusted life years) cioè anni di vita guadagnati ponderati per la qualità della vita per due diverse tecniche dialitiche: emodialisi standard HD ed emodiafiltrazione HDF.

Osservando i dati emersi dallo studio ci siamo resi conto che i due gruppi percepiscono la qualità della loro vita in maniera molto simile, nonostante i parametri dialitici ed ematologici, oggetto di studio, siano significativamente migliori nel gruppo in trattamento dialitico con la metodica dell'Emodiafiltrazione.

Parole chiave: Health Technology Assessment, QALYs, Analisi Costo-Utilità, Qualità percepita

Indirizzo degli Autori:

Umbero Tulli

UOC Nefrologia e Dialisi

Ospedale di Tivoli

Roma

umtulli@tin.it 


\section{Bibliografia}

1. Apolone G., Mingardi G., Mosconi P. Misurare la salute in nefrologia e dialisi. Roma: Pensiero Scientifico, 2000.

2. Claxton K., Lacey L., Walker S. Selecting treatments: a decision theoretic approach. Journal of the Royal Statistical Society A 2000; 163: 211-25.

3. Drummond M, O'Brien B, Stoddar G, Torrance G. Methods for the Economic Evaluation of Health Care Programmes. London (UK): Oxford University Press, 1997.

4. Gold M, Siegel J, Russel L, Weinstein M. Cost effectiveness in health and medicine. New York, NY: Oxford University Press, 28-1.
5. Drummond M. Evidence-based medicine and cost-effectiveness: uneasy bedfellows [EBM Note]. Evidence-Based Medicine 1998; 3: 133.

6. Elixhauser A, Halpern M, Schmier J, Luce BR. Health care CBA and CEA from 1991 to 1996: an updated bibliography. Med Care 1998; 36 (Suppl 5): MS1-9.

7. O'Brien B. Principles of economic evaluation for health care programs. J Rheumatol 1995; 22: 1399-402.

8. Zarnke KB, Levine MA, O'Brien BJ. Cost-benefit analyses in the health care literature: don't judge a study by its label. J Clin Epidemiol 1997; 50: 813-22.

9. $\mathrm{http} / / /$ marionegri.it/qdv/questionari/sf36/sf36v1 